\title{
A Woman With Chest Pain, Syncope, and Transaminitis
}

\author{
Ankitkumar K. Patel, MD, MPH, and Brendan O'Hare, MD
}

\section{Case Presentation}

The patient is a 49 year-old female with past medical history of anxiety and hyperlipidemia who presented to an outside hospital with complaints of five hours of substernal chest pain followed by three episodes of syncope witnessed by her son. At presentation in the emergency department the patient denied any current chest pain or shortness of breath. She received $325 \mathrm{mg}$ of aspirin en route to the hospital by EMS. Her vital signs were temperature $100^{\circ}$ Fahrenheit, heart rate 60 beats/minute, blood pressure $101 / 50 \mathrm{~mm} \mathrm{Hg}$, respiratory rate 20 breaths/minute, and a pulse oxygenation of $98 \%$ on room air. The patient's EKG showed ST elevations in the inferior leads. The patient's laboratory studies were: white blood cell (wbc) count $14 \mathrm{~B} / \mathrm{L}$, hemoglobin $13.2 \mathrm{~g} /$ $\mathrm{dL}$, platelets $153 \mathrm{~B} / \mathrm{L}, \mathrm{CKMB} 32 \mathrm{U} / \mathrm{L}$, troponin $8.27 \mathrm{ug} / \mathrm{L}$, and $\mathrm{CK} 24.5 \mathrm{U} / \mathrm{L}$. The patient was started on intravenous heparin and integrillin drips and transferred to Jefferson for emergent cardiac catheterization.

The patient had left and right coronary angiography which showed no evidence of occlusive coronary disease. Left heart catheterization demonstrated moderate to severe inferoapical hypokinesis and a mildly depressed left ventricular ejection fraction (40\%). During the procedure, the patient was noted to have 2:1 AV heart block with intraventricular conduction delay, and a temporary transvenous pacemaker was placed. The patient was then transferred to the cardiac care unit.

In the cardiac care unit, the patient reported that she had no known drug allergies. Her only outpatient medication was a statin that was started six weeks ago. The patient denied any past surgical history. The patient consumed alcohol socially, had a 15 pack year history of smoking, and no history of illicit drug use. The patient's father died of a myocardial infarction at age 62 , and the patient's mother was alive with a history of diabetes mellitus and arrhthymia. The patient noted that she had some nasal congestion for two days prior to the chest pain and syncope. The patient also reported working in her garden with rose bushes and was certain that she had a neck rash due to poison oak. Lastly, the patient reported decreased oral intake for the past two days due to "flu-like" disease.

Overnight, the patient had multiple episodes of bradycardia that warranted venous pacing on telemetry. Significant laboratory studies were: wbc $4.3 \mathrm{~B} / \mathrm{L}$, AST $539 \mathrm{U} / \mathrm{L}$, ALT 313 $\mathrm{U} / \mathrm{L}$, and troponin $14.7 \mathrm{ug} / \mathrm{L}$. The patient underwent successful implantation of a dual chamber VDD pacemaker. A transthoracic echocardiogram demonstrated an ejection fraction of $60 \%$, mild to moderate mitral regurgitation, and an inferior vena cava normal in size without inspiratory collapse. The patient was transferred to the telemetry floor service.

On the third hospital day, the patient had no overnight complaints and wanted to be discharged home. Significant laboratory studies were a troponin of 16.5 and AST $299 \mathrm{U} / \mathrm{L}$, and ALT $250 \mathrm{U} / \mathrm{L}$. The patient had a CT of her head which showed no fracture or bleed. A CT of her chest demonstrated multifocal pneumonia with bilateral pleural effusions and subcentimeter pulmonary nodules. The patient was diagnosed with pneumonia and was started on ceftriaxone, azithromycin and flagyl.

On the fourth hospital day, the patient had an overnight fever of $100.7^{\circ}$ Fahrenheit and an episode of oxygen desaturation to $89 \%$ on 2 liters oxygen via nasal cannula. Significant laboratory studies were: wbc $14.9 \mathrm{~B} / \mathrm{L}$, troponin $14.1 \mathrm{ug} / \mathrm{L}$, AST $247 \mathrm{U} / \mathrm{L}$, and ALT $240 \mathrm{U} / \mathrm{L}$.

On the morning of the fifth hospital day, the patient had a fever of $100.6^{\circ}$ Fahrenheit and an episode of tachycardia with oxygen desaturation to $75 \%$ on 2 liters with an increased respiratory rate (18 to 28 breaths/minute). An arterial blood gas showed pH 7.50, pCO2 23, PO2 138, HCO3 18, O2 sat 99\%. Significant laboratory studies were wbc $18.9 \mathrm{~B} / \mathrm{L}$, troponin $8.9 \mathrm{ug} / \mathrm{L}$, AST $279 \mathrm{U} / \mathrm{L}$, ALT $288 \mathrm{U} / \mathrm{L}$, lactate $40.8 \mathrm{mmol} / \mathrm{L}$, and BNP 2058. The patient was treated with nebulizer treatments, intravenous furosemide, and an anxiolytic. A transthoracic echocardiogram demonstrated an ejection fraction of $25 \%$, moderate-severe mitral regurgitation, severely decreased left ventricular systolic function with segmental wall motion abnormalities, mild right atrial enlargement, dilated inferior vena cava, and right ventricular enlargement with decreased function.

Later in the afternoon, the patient had complaints of shortness of breath and increased anxiety. Vital signs at the time were heart rate $123 \mathrm{bpm}$, blood pressure $100 / 60 \mathrm{~mm} \mathrm{Hg}$, respiratory rate 36 breaths/minute, pulse oxygenation of $90 \%$ on $100 \%$ nonrebreather mask. An arterial blood gas demonstrated $\mathrm{pH} 7.49$, pCO2 27, PO2 60, HCO3 20, O2 sat 93\%. The patient was once again treated with nebulizer treatments, intravenous lasix and had symptomatic relief. Three hours later, the patient had worsening respiratory distress and was found to have a respiratory rate of $48-60$ with a pulse oxygenation of $68 \%$ on room air and $88 \%$ on $100 \%$ non-rebreather mask. A rapid response was called for worsening respiratory distress.

The patient was transferred to the cardiac care unit and emergently intubated. Subsequently, the patient became progressively hypotensive and a central line was placed, and intravenous norepinephrine was started. The patient was presumed to have worsening sepsis secondary to multifocal pneumonia with cardiomyopathy of sepsis. Antibiotic coverage was broadened to vancomycin and zosyn. In addition, doxycycline was added to cover possible ricketsial infections. A complete rheumatologic and autoimmune work up was sent for laboratory studies.

The following day, the patient had a transesophageal echocardiogram which showed no vegetations. Azithromycin was added to cover atypical organisms, and the patient was started 
on an acute respiratory distress syndrome ventilator protocol. Significant laboratory studies were: wbc $23.1 \mathrm{~B} / \mathrm{L}$ with $6 \%$ bands, AST $1209 \mathrm{U} / \mathrm{L}$, ALT $873 \mathrm{U} / \mathrm{L}$, and troponin 6.29 ug/L.

On the seventh day, the patient underwent fiberoptic bronchoscopy which was grossly normal and bronchoalveolar lavage of the right upper lobe was sent for cultures. Significant laboratory studies were: wbc $19.1 \mathrm{U} / \mathrm{L}$, creatinine $1.5 \mathrm{umol} / \mathrm{L}, \mathrm{AST}$ $2220 \mathrm{U} / \mathrm{L}$, and ALT $1516 \mathrm{U} / \mathrm{L}$. The patient became progressively more hypotensive, and the norepinephrine drip was titrated. The patient also had decreasing urine output and worsening transaminitis was thought to be due to ischemic hepatitis.

On the eighth day, the patient developed a supraventricular tachycardia which was treated with adenosine and revealed an underlying atrial flutter rhythm. The patient was started on digoxin for rate control. With worsening hypotension and tachycardia, the patient was started on phenylephrine with plans to wean down norepinephrine. Due to worsening clinical status, Xigris therapy was also initiated. A transthoracic echocardiogram showed an ejection fraction of $10 \%$, moderatesevere mitral regurgitation, severe left ventricular systolic function (with only the basal-inferolateral segment contracting - the remainder was hypokinetic/akinetic), right ventricular enlargement, moderate-severe tricuspid regurgitation. Late in the evening, the patient's clinical exam had a significant change with cool extremities and hypothermia. Laboratory studies were consistent with a state of disseminated intravascular coagulation and worsening renal function. A family meeting was called to discuss the worsening clinical status and overall prognosis. Cardiology consultation offered right heart catheterization and the placement of an intraortic balloon pump (IABP). The right heart catheterization showed HR 130, aortic pressure 91/59, PA 55/39 mean 45, wedge 54, RA 50/49 mean 40, RV 55/39, SVR 750, PVR 1126, CO by Fick 3.20, CI by Fick 1.59. The patient was diagnosed with cardiogenic shock, and the IABP was set at 1:1 and a Shiley catheter was placed for urgent continuous veno-venous hemodialysis. The patient was weaned off of the phenyephrine and started on dobutamine, dopamine, and eventually epinephrine.

On the morning of the ninth hospital day, the cardiac care unit, heart failure, pulmonary, renal, and cardiothoracic surgery teams discussed the patient's case. Significant laboratory studies were: wbc $21 \mathrm{U} / \mathrm{L}$, hemoglobin $7.9 \mathrm{~g} / \mathrm{dL}$, platelets $59 \mathrm{~B} /$ $\mathrm{L}$, creatinine $2.5 \mathrm{umol} / \mathrm{L}$, and AST $12217 \mathrm{U} / \mathrm{L}$, and ALT $4060 \mathrm{U} /$ L. Due to the rapid and progressive decline in cardiac function due to severe myocarditis, a decision was made to place a right ventricular assist device (RVAD) and a left ventricular assist device (LVAD), commonly called a BiVAD. The patient underwent surgery with the RVAD connecting the right atrium to the pulmonary arteries and the LVAD connecting the left ventricle to the aorta. A cardiac myocardial biopsy was also conducted during the surgical procedure. A collection of all lab results is included in Table 1.

\section{Discussion}

Often, myocarditis is an under-diagnosed medical condition. Its pathophysiology is marked by a triad: cardiac injury by inciting agent, immunologic response, and inflammation. Generally speaking $1 / 3^{\text {rd }}$ of patients recover, $1 / 3^{\text {rd }}$ experience permanent cardiac dysfunction, and $1 / 3^{\text {rd }}$ deteriorate to either death or cardiac transplant.

Myocarditis' true incidence is most likely under represented. It has been postulated that up to $8.6 \%$ of sudden cardiac deaths may be attributable to myocarditis. ${ }^{1}$ Up to $10 \%$ to $40 \%$ of what was thought to be idiopathic dilated cardiomyopathy in children may in fact be occult myocarditis. ${ }^{2}$ As diagnostic techniques such as immunohistochemistry, electron microscopy, and molecular proteomics advance inevitably the incidence of myocarditis will rise.

The diagnosis most often implies viral infection. The most common vectors of disease have thought to be enteroviruses such as coxsackie or adenovirus. However, any virus can cause myocarditis: cytomegalovirus, parvovirus, influenza, human immunodeficiency virus, hepatitis $\mathrm{C}$, and Epstein-Barr virus have all been implicated. Furthermore, tricyclic antidepressants, clozapine, the smallpox vaccine, radiation, heat stroke, and hypothermia can all cause inflammation of cardiac tissue.

Clinically, the onset of symptoms can be insidious or rapid. Acutely, a viral prodrome often predominates with signs including fatigue, arthralgias, and fever. After this, cardiac manifestations may develop. In this particular case our patient presented with arrhythmia and heart block, which can be seen as the presenting symptom in $5 \%$ of cases. ${ }^{3}$ Other symptoms such as chest pain and heart failure are often also present. Unfortunately, some patients may present with hemodynamic compromise and a fulminant picture.

Laboratory testing can include elevated ESR, WBC, or troponin. EKG findings are often non-specific and may range from subtle abnormalities to ST elevations. One study revealed that in the setting of an acute myocardial infarction and normal cardiac catheterization, $38 \%$ of patients were thought to have myocarditis. ${ }^{4}$ Non-invasive techniques include transthoracic echocardiography in which regional wall motion abnormalities are typically more common than cardiac dilation. This is especially true in the acute to sub-acute period before cardiac remodeling has occurred. A less common used modality is indium-111 anti-myosin imaging. This is a nuclear medicine study in which anti-myosin antibodies are introduced, they bind to injured myocardium, and the results can then be quantified. The technique is excellent for detecting damage; however, distinguishing between a myocardial infarction and myocarditis may be difficult. In fact, the sensitivity is good at $85 \%$ to $100 \%$, but the specificity is only $55 \% .{ }^{5}$ Another option 


\begin{tabular}{|c|c|c|}
\hline Infectious & Cardiovascular & Hepatology \\
\hline Legionella $\mathrm{Ag}$ negative & Cholesterol 190 & Hep A IgG/IgM negative \\
\hline Lyme disease Ab 0.12 (negative) & HDL 33 & Hep B IgG/lgM negative \\
\hline Lyme PCR negative & LDL 120 & Hep B core Ab negative \\
\hline M.Pneumoniae IgM negative & Triglycerides 187 & Hep B surface Ab negative \\
\hline Ehrlichia Ab lgG/lgM negative & & Hep C Ab negative \\
\hline RPR negative & Rheumatology & Hep C PCR negative \\
\hline HIV negative & RF 21 (wnl) & Alpha-1 antitryp quant 251 (high) \\
\hline EBV IgM negative & ACE 21 (wnl) & Copper 47.7 (high) \\
\hline EBV IgG positive & ANA negative & \\
\hline EBV nuclear Ab positive & anti dsDNA 11 (wnl) & Hematology \\
\hline CMV negative & ASM negative & Serotonin release negative \\
\hline Herpes negative & C-ANCA negative & PLT factor 4 negative \\
\hline RSV negative & P-ANCA negative & Iron 52 (wnl) \\
\hline Varicella IgG Ab reactive & & TIBC 252 (low) \\
\hline VZV IgM 0.16 (negative) & Endocrinology & FeSat 24\% (low-normal) \\
\hline Coxsackie types negative & $\mathrm{HgbA} 1 \mathrm{C} 5.7 \%$ & Ferritin 1085 (high) \\
\hline Ent-virus PCR negative & TSH 0.65 (wnl) & \\
\hline Influenza $A+B$ negative & Cortisol 94 & \\
\hline \multicolumn{3}{|l|}{ CMV Ab IgM negative } \\
\hline Toxoplasma negative & & \\
\hline
\end{tabular}

is cardiac MRI which uses contrast kinetics to identify areas of damage and may be able to differentiate myocardial infarction from myocarditis. Epicardial predominance, a non-contiguous pattern, and involvement of the lateral wall are more common in myocarditis according to one study. ${ }^{6}$

The gold standard in the diagnosis of myocarditis is biopsy. For 20 years, the Dallas criteria have defined a positive biopsy as one that displays inflammatory cells plus necrosis. A borderline biopsy is one that has inflammatory cells but no necrosis. ${ }^{7}$ However, this system is imperfect. The nature of myocarditis is such that it does not involve the entire myocardium. As such, even if one takes five biopsy specimens, the diagnosis will be missed $33 \%$ of the time. ${ }^{8}$ Moreover, the disease often affects the lateral wall which is difficult to biopsy using current techniques. Detection of myocarditis via pathology can also be subject to observer differences. Furthermore, there are risks involved with the procedure such as vascular access issues, arrhythmias, and perforation.

Prognosis generally depends on the type of myocarditis. For example, giant cell myocarditis usually progresses quite rapidly, while other forms progress more subtly. As stated previously, patients may recover completely, sub-acutely progress to permanent cardiac dysfunction, or rapidly deteriorate to death or transplant. Generally, there is a $20 \%$ mortality at one year and $50 \%$ at four years. Predictors of death or requiring transplantation in the future include the presenting symptom of syncope or bundle branch block, an ejection fraction of $<40 \%$, and persistence of viral genome in the myocardium. ${ }^{9}$ Unfortunately, our patient had two of these three predictors.

Regrettably, the treatment options for myocarditis leave much to be desired. The first steps are supportive therapies such as ACE inhibitors, diuretics, and beta blockers when clinically stable. The National Institutes of Health conducted a trial in which they randomized standard heart failure therapy versus steroids + azathioprine versus steroids + cyclosporine for six months. The results showed no difference in ejection fraction or mortality after four years. ${ }^{10}$ There was some improvement during the acute treatment phase, but this was transient. Final recommendations included considering steroids in giant cell myocarditis or in hemodynamic compromise. In another trial, 
interferon was used to treat 22 patients for six months. Virus was eliminated from myocardium in all 22 patients and 15 of 22 showed improvement in left ventricular systolic function. ${ }^{11}$ The results were fairly promising and, consequently, have spurred an ongoing phase III trial. IVIG has been sought as therapy due to its effects on auto-antibodies. In 2003, IVIG was compared to placebo. Systolic function improved from $25 \%$ at baseline to $41 \%$ at six months, but there was no statistical difference in the two arms of the trial. ${ }^{12}$ Currently, the most recognized treatments are supportive. Specifically, left ventricular assist devices have been shown to be beneficial in select patients. Indications for LVAD include: cardiac index $<2.0 \mathrm{~L} / \mathrm{min} / \mathrm{m} 2$, central venous pressure or left atrial pressure $>20$, and urine output $<20 \mathrm{~mL} /$ hour despite maximal pharmacological therapy. ${ }^{13}$ Furthermore, the disease may be so progressive that both right and left ventricular assist devices may be needed, such as in this case presented here. Indications for a $\mathrm{BiVAD}$ are renal, hepatic, and pulmonary dysfunction, seen with our patient. ${ }^{14}$

\section{References}

1. Fabre A, Sheppard MN. Sudden adult death syndrome and other non-ischemic causes of sudden cardiac death. Heart 2006. 92:316-320.

2. Nugent AW, Piers EF, Chondros P, Carlin JB, Cheung M, Wilkinson LC, Davis AM, Kahler SG, Chow CW, Wilkinson JL, Weintraub RG. The epidemiology of childhood cardiomyopathy in Australia. NEJM 2003. 348(17): 1639-1646

3. Cooper LT, Berry GJ, Shabetai R. Idiopathic giant-cell myocarditis - natural history and treatment. NEJM 1997. 336(26):1860-1866.

4. Sarda L, Colin P, Boccara F, Daou D, Lebtahi R, Faraggi M, Nguyen C, Cohen A, Slama MS, Steg PG, Le Guludec D. Myocarditis in patients with clinical presentation of myocardial infarction and normal coronary angiograms. JACC 2001. 37(3): 786-792.

5. Narula J, Khaw BA, Dec GW Jr, Palacios IF, Southern JF, Fallon JT, Strauss HW, Haber E, Yasuda T. Brief report: recognition of acute myocarditis masquerading as acute myocardial infarction. NEJM 1993, 328(2):100-104.
6. Codreanu A, Djaballah W, Angioi M, Ethevenot G, Moulin F, Felblinger J, Sadoul N, Karcher G, Aliot E, Marie PY. Detection of myocarditis by contrastenhanced MRI in patients presenting with acute coronary syndrome but no stenosis. Jour of MRI 2007. 25(5): 957-964.

7. Aretz HT, Billingham ME, Edwards WD, Factor SM, Fallon JT, Fenoglio JJ Jr, Olsen EG, Schoen FJ. Myocarditis: a histopathologic definition and classification. Am J Cardiovasc Patho. 1987. 1: 3-14

8. Mahrholdt H, Goedecke C, Wagner A, Meinhardt G, Athanasiadis A, Vogelsberg H, Fritz P, Klingel K, Kandolf R, Sechtem U. Cardiovascular magnetic resonance assessment of human myocarditis: a comparison to histology and molecular pathology. Circulation 2004. 109:1250-1258.

9. Mann DL. Determinants of myocardial recovery in myocarditis has the time come for molecular fingerprinting? JACC 2005. 46(6):1043-4.

10. Mason JW, O’Connell JB, Herskowitz A, Rose NR, McManus BM, Billingham ME, Moon TE. A clinical trial of immunosuppressive therapy for myocarditis. The Myocarditis Treatment Trial Investigators. NEJM 1995. 333(5):269-75.

11. Kühl U, Pauschinger M, Schwimmbeck PL, Seeberg B, Lober C, Noutsias M, Poller W, Schultheiss HP. Interferon-beta treatment eliminates cardiotropic viruses and improves left ventricular function in patients with myocardial persistence of viral genomes and left ventricular dysfunction. Circulation 2003. 107(22):2793-2798

12. McNamara DM, Holubkov R, Starling RC, Dec GW, Loh E, Torre-Amione G, Gass A, Janosko K, Tokarczyk T, Kessler P, Mann DL, Feldman AM. Controlled trial of intravenous immune globulin in recent-onset dilated cardiomyopathy. Circulation 2001. 103(18):2254-9.

13. Reiss N, el-Banayosy A, Posival H, Morshuis M, Minami K, Körfer R. Management of acute fulminant myocarditis using circulatory support systems. Artif Organs 1996. 20(8):964-70.

14. Farrar DJ, Holman WR, McBride LR, Kormos RL, Icenogle TB, Hendry PJ, Moore CH, Loisance DY, El-Banayosy A, Frazier H. Long-term follow-up of Thoratec ventricular assist device bridge-to-recovery patients successfully removed from support after recovery of ventricular function. J Heart Lung Transplant 2002. 21(5):516-21. 